\title{
Developments towards in-gas-jet laser spectroscopy studies of actinium isotopes at LISOL
}

\author{
S. Raeder ${ }^{\mathrm{a}, \mathrm{b}, \mathrm{c}, *}$, B. Bastin ${ }^{\mathrm{d}}$, M. Block ${ }^{\mathrm{b}, \mathrm{c}, \mathrm{f}}$, P. Creemers ${ }^{\mathrm{a}}$, P. Delahaye ${ }^{\mathrm{d}}$, R. Ferrer ${ }^{\mathrm{a}}$, X. Fléchard $^{\mathrm{e}}$, S. Franchoo $^{\mathrm{g}}$, \\ L. Ghys ${ }^{\mathrm{a}, \mathrm{l}}$, L.P. Gaffney ${ }^{\mathrm{a}, 1}$, C. Granados ${ }^{\mathrm{a}}$, R. Heinke ${ }^{\mathrm{h}}$, L. Hijazi ${ }^{\mathrm{d}}$, M. Huyse ${ }^{\mathrm{a}}$, T. Kron ${ }^{\mathrm{h}}$, Yu. Kudryavtsev ${ }^{\mathrm{a}}$, \\ M. Laatiaoui ${ }^{\text {b,c }}$, N. Lecesne ${ }^{\mathrm{d}}$, F. Luton ${ }^{\mathrm{d}}$, I.D. Moore ${ }^{\mathrm{i}}$, Y. Martinez ${ }^{\mathrm{a}}$, E. Mogilevskiy ${ }^{\mathrm{a}, \mathrm{j}}$, P. Naubereit ${ }^{\mathrm{h}}$, J. Piot ${ }^{\mathrm{d}}$, \\ S. Rothe ${ }^{k}$, H. Savajols ${ }^{d}$, S. Sels ${ }^{a}$, V. Sonnenschein ${ }^{i}$, E. Traykov ${ }^{d}$, C. Van Beveren ${ }^{a}$, P. Van den Bergh ${ }^{\text {a }}$, \\ P. Van Duppen ${ }^{\mathrm{a}}$, K. Wendt ${ }^{\mathrm{h}}$, A. Zadvornaya ${ }^{\mathrm{a}}$
}

${ }^{a}$ KU Leuven, Instituut voor Kern- en Stralingsfysica, Celestijnenlaan 200D, B-3001 Leuven, Belgium

${ }^{\mathrm{b}}$ Helmholtz-Institut Mainz, 55128 Mainz, Germany

${ }^{\mathrm{C}}$ GSI Helmholtzzentrum für Schwerionenforschung GmbH, Planckstraße 1, 64291 Darmstadt, Germany

${ }^{\mathrm{d}}$ GANIL, CEA/DSM-CNRS/IN2P3, B.P. 55027, 14076 Caen, France

e LPC Caen, ENSICAEN, Université de Caen, CNRS/IN2P3, Caen, France

${ }^{\mathrm{f}}$ Institut für Kernchemie, Johannes Gutenberg Universität, 55128 Mainz, Germany

${ }^{\mathrm{g}}$ Institute de Physique Nucléaire (IPN) d'Orsay, 91406 Orsay, Cedex, France

${ }^{\mathrm{h}}$ Institut für Physik, Johannes Gutenberg Universität, 55128 Mainz, Germany

${ }^{i}$ Department of Physics, University of Jyväskylä, P.O. Box 35 (YFL), Jyväskylä, FI40014, Finland

${ }^{\mathrm{j}}$ Faculty of Mechanics and Mathematics, Lomonosov Moscow State University, Leninskie gory, 1, 119992 Moscow, Russia

${ }^{\mathrm{k}} \mathrm{CERN}, \mathrm{CH}-1211$ Genève, Switzerland

${ }^{1}$ SCK-CEN, Belgian Nuclear Research Center, Boeretang 200, 2400 Mol, Belgium

\section{A R T I C L E I N F O}

\section{Article history:}

Received 6 September 2015

Received in revised form 20 November 2015

Accepted 10 December 2015

Available online 4 February 2016

\section{Keywords:}

Resonance ionization spectroscopy

Gas cell

Gas jet

Actinium

\begin{abstract}
A B S T R A C T
To study exotic nuclides at the borders of stability with laser ionization and spectroscopy techniques, highest efficiencies in combination with a high spectral resolution are required. These usually opposing requirements are reconciled by applying the in-gas-laser ionization and spectroscopy (IGLIS) technique in the supersonic gas jet produced by a de Laval nozzle installed at the exit of the stopping gas cell. Carrying out laser ionization in the low-temperature and low density supersonic gas jet eliminates pressure broadening, which will significantly improve the spectral resolution. This article presents the required modifications at the Leuven Isotope Separator On-Line (LISOL) facility that are needed for the first on-line studies of in-gas-jet laser spectroscopy. Different geometries for the gas outlet and extraction ion guides have been tested for their performance regarding the acceptance of laser ionized species as well as for their differential pumping capacities. The specifications and performance of the temporarily installed high repetition rate laser system, including a narrow bandwidth injection-locked Ti:sapphire laser, are discussed and first preliminary results on neutron-deficient actinium isotopes are presented indicating the high capability of this novel technique.
\end{abstract}

(c) 2016 Elsevier B.V. All rights reserved.

\section{Introduction}

At the Leuven Isotope Separator On-Line (LISOL) facility the ingas laser ionization and spectroscopy (IGLIS) technique is used to study short-lived nuclides produced in heavy-ion fusion reactions

\footnotetext{
* Corresponding author at: GSI Helmholtzzentrum für Schwerionenforschung GmbH, Planckstraße 1, 64291 Darmstadt, Germany.

E-mail address: s.raeder@gsi.de (S. Raeder).

1 Present address: School of Engineering, University of the West of Scotland, Paisley PA1 2BE, United Kingdom.
}

where highly energetic projectiles impinge on thin solid targets. This technique was previously employed within the highpressure environment of the stopping gas cell to study ground and isomeric state properties of neutron-deficient ${ }^{57-59} \mathrm{Cu}[1]$ and ${ }^{97-101} \mathrm{Ag}[2]$ isotopes by resonant laser excitation and ionization. To improve the spectral resolution (typically $4-10 \mathrm{GHz}$ due to pressure broadening) and the selectivity of this technique, offline studies on resonant ionization in the low-temperature and low-density regime of the supersonic free jet formed at the gas cell exit hole were successfully performed using stable $\mathrm{Cu}$ isotopes [3]. 
Here a resolution down to $450 \mathrm{MHz}$ for the $327 \mathrm{~nm}$ optical ground state transition in Cu could be demonstrated using pulsed amplification of a cw diode laser an improvement of one order of magnitude compared to in-gas-cell spectroscopy. To explore the full capability of the in-gas-jet ionization technique a new off-line laboratory is being commissioned at KU Leuven [4]. Finally, this technique will be used at the low energy front-end of the $S^{3}$ in-flight separator at GANIL [5] to study rare isotopes at the extremes of existence. This technique was now applied for the first time online at the LISOL facility to study neutron-deficient actinium isotopes including the semi-magic ${ }^{215}$ Ac nucleus.

The first attempt to perform resonant laser ionization in the gas cell on actinium isotopes was reported in [5]. This research was motivated by studying the evolution of the $N=126$ shell closure towards heavier $Z$ systems by investigating electrical quadrupole and magnetic dipole moments, as well as by the investigation on shape effects occurring in this region of the nuclear chart through the observation of changes in charge radii. Complementary, neutron-rich actinium isotopes, located in a region with expected octupole deformation [6], are accessible at ISOL facilities [7]. Atomic levels of actinium for resonant excitation are known from the measurements of arc and hollow-cathode spectra using longlived ${ }^{227} \mathrm{Ac}[8,9]$, while investigations on the first ionization potential (IP) as well as on auto-ionizing (AI) resonance above the first IP have been carried out in a previous work within the collaboration [10]. Additional spectroscopic off-line studies focused on the identification of levels featuring a wide hyperfine structure (HFS) splitting with a suitable transition at $438 \mathrm{~nm}$ [11]. Using this transition, the HFS splitting of the neutron deficient actinium isotopes ${ }^{212-215}$ Ac was partly resolved in in-gas-cell laser spectroscopic studies at LISOL [12]. Nevertheless, the spectral resolution of about $6 \mathrm{GHz}$ did not allow the extraction of all required parameters with an adequate precision. Especially the information on the spectroscopic quadrupole moment and therefore the nuclear deformation could not be determined.

The in-gas-jet ionization technique overcomes these restrictions and the production rates of actinium isotopes made an online application at LISOL feasible. For the investigations on rare isotopes the total efficiency of the method is crucial as the production rates are small. During the previous off-line tests on copper reported in [3] the total efficiency could not be estimated due to the unknown evaporation rate of stable $\mathrm{Cu}$ from the filament. However, the duty cycle when using the existing $200 \mathrm{~Hz}$ Excimer-pumped dye laser system for ionization in the fast propagating gas jet as well as the large divergence angle of the freely expanding jet limit the potential efficiency. The existing frontend at LISOL furthermore constrained the implementation of this technique limiting the final performance.

This article summarizes the preparation work for the on-line application of the in-gas-jet ionization involving the gas-jet formation, the ion extraction as well as the performance and characteristics of the high-repetition laser system that was installed at the LISOL facility to improve the total performance. The first preliminary results will also be summarized here.

\section{Production, ion extraction and interaction region}

At LISOL the radioactive isotopes are produced using the fusion reaction from ${ }^{20,22} \mathrm{Ne}$ projectiles $(0.16 \mathrm{p} \mu \mathrm{A}, 145 \mathrm{MeV})$ onto a $1 \mu \mathrm{m}$ thick ${ }^{197} \mathrm{Au}$ target, with production cross sections as low as 1.6 mbarn for ${ }^{212} \mathrm{Ac}[5,13]$. The target is located inside a gas cell filled with about 500 mbar of argon for thermalization and neutralization of the reaction products. The neutralized products are guided by the gas flow towards the exit nozzle of the gas cell. Remaining charged particles are removed by an electrostatic ion

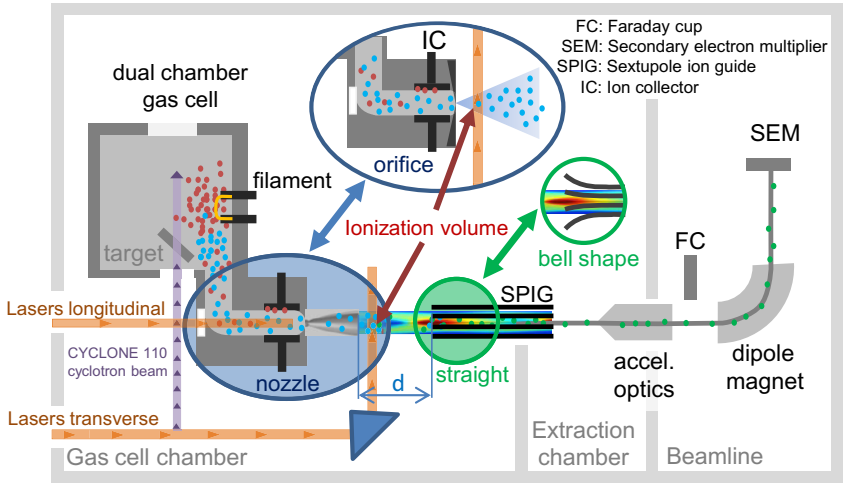

Fig. 1. Schematic diagram of the experimental set-up at LISOL illustrating different options investigated in preparation for the on-line studies. In-gas-cell (longitudinal) and in-gas-jet (transverse) ionization for the off-line (filament) and on-line measurements are indicated. The two options under investigation for the gas cell exit, a free jet from an orifice and a shaped gas jet from a de Laval nozzle are depicted in the blue circled areas. The green circled area indicates the tested SPIG configurations for an enhanced ion capture efficiency. (For interpretation of the references to color in this figure legend, the reader is referred to the web version of this article.)

collector. The neutralized atoms are ionized using resonant laser ionization in a two-step ionization scheme [14] allowing for an improved selectivity in radioactive ion beam (RIB) production [15] as well as for laser spectroscopic investigations.

For the laser ionization in the gas jet a high repetition rate laser system as well as an extended laser-atom ionization region are required for highest efficiencies $[3,4]$. As the argon jet velocity is about $550 \mathrm{~m} / \mathrm{s}$ an ionization region of $55 \mathrm{~mm}$ length is needed assuming a laser repetition rate of $10 \mathrm{kHz}$. The schematic setup for the experiment in combination with investigated options is depicted in Fig. 1. Using the dual-chamber gas cell [16] the gas cell chamber and the position of the primary cyclotron beam restrict the distance between gas cell exit and the beginning of the sextupole ion guide (SPIG) to about $22 \mathrm{~mm}$. The SPIG is required for ion extraction and differential pumping between the gas cell chamber and the extraction chamber. To test the capturing efficiency of the photo ions in the gas jet by the SPIG two different options for the gas outlet at the gas cell exit were tested. The standard configuration is a simple orifice with a hole diameter of $0.8 \mathrm{~mm}$, leading to a free expanding gas jet. For in-gas-cell ionization the SPIG is located within a few $\mathrm{mm}$ after the orifice to ensure an efficient capturing while a DC gradient is applied to dissociate molecules formed during the ion transport in the gas cell. The second option is a de Laval nozzle with a throat diameter of $1 \mathrm{~mm}$ as indicated in the blue encircled areas in Fig. 1. The gas dynamic calculations indicate that the argon boundary layer inside the nozzle limits the effective Mach number of $M \approx 5.5$ and a jet diameter of about $3.5 \mathrm{~mm}$.

Fig. 2 shows the measured extraction efficiency for ions created inside the gas cell and transported to the first Faraday cup (FC) after acceleration as a function of the distance for both options. For these measurements stable $\mathrm{Cu}$ isotopes were evaporated from a filament and were ionized inside the gas cell using an Excimerpumped (LPX 240i, Lambda Physik) dye laser system (Scanmate, Lambda Physik) at a repetition rate of $50 \mathrm{~Hz}$ [17]. The extraction efficiency for the free jet clearly drops when the distance $d$ (see Fig. 1) between orifice and SPIG is increased, limiting the effective distance to values below $10 \mathrm{~mm}$. The extraction efficiency for the de Laval nozzle shows a better performance and is constant at the level of about $80 \%$ within the range of the possible nozzle SPIG distance, indicating that the gas jet is more forward directed compared to the free-expanding gas jet from the orifice. In contrast to the low divergence shape of the jet from the de Laval nozzle an 


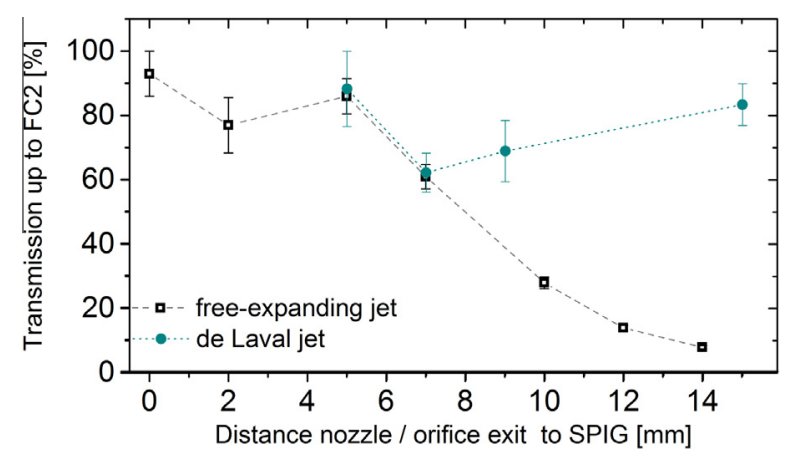

Fig. 2. Dependence of the transmission of in-gas-cell ionized stable $\mathrm{Cu}$ isotopes on the distance $d$ for the two options of gas jets. The FC signal is referenced to the ion current captured directly onto the SPIG rods. The points are connected to guide the eyes.

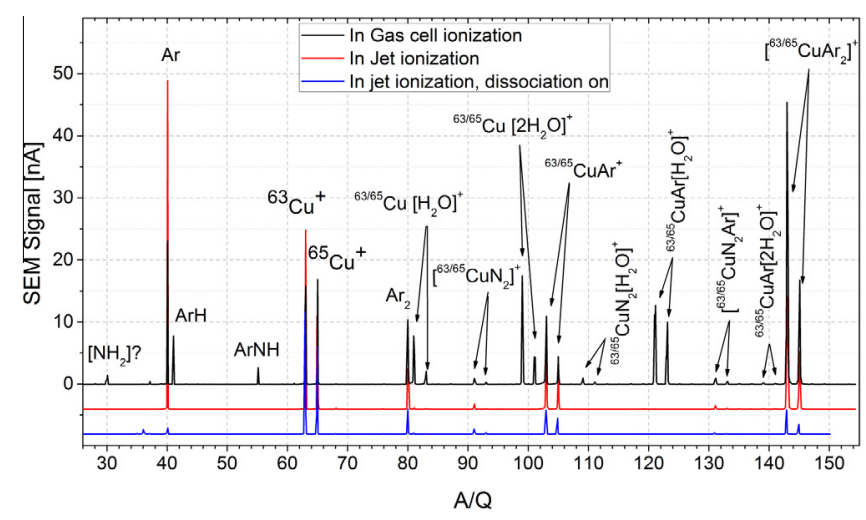

Fig. 3. Mass spectra obtained from in-gas-cell (black curve) and in-gas-jet ionization (red curve) show the molecular formation of $\mathrm{Cu}$ ions. The blue curve represents the case of in-gas-jet ionization in combination with a SPIG offset voltage of $300 \mathrm{~V}$ for CID. The red and blue curves are shifted for visibility. (For interpretation of the references to color in this figure legend, the reader is referred to the web version of this article.)

opening angle of about $30^{\circ}$ was estimated for the free jet in off-line experiments [3]. This conclusion is supported by the observations from the differential pumping capability of the SPIG. In contrast to the orifice, a larger gas cell-SPIG distance is required for the de Laval nozzle in order to maintain a working pressure below $1 \cdot 10^{-4}$ mbar in the extraction chamber when operating the gas cell at the nominal pressure of 350 mbar, which is required to efficiently stop the actinium fusion products. Additionally, the ratio of in-gas-cell to in-gas-jet ionized $\mathrm{Cu}$ was determined to estimate the efficiency performance. For a laser beam diameter of $5 \mathrm{~mm}$ irradiating the gas jet in a transversal arrangement for both laser beams and laser repetition rate of $100 \mathrm{~Hz}$ a ratio of about 1000 is roughly expected from duty cycle and geometrical considerations. For the orifice a ratio of 500 was observed while for the de Laval nozzle a ratio below 200 was repeatedly measured. This exceeds the expectations by a factor of $4-5$ which could be explained by a reduction of collisional de-excitation compared to the high pressure environment inside the gas cell. In summary the available de Laval nozzle, although not fully characterized and optimized, showed a superior performance in comparison to the orifice. The length of the diverging part of the de Laval nozzle is $10.5 \mathrm{~mm}$. This limits the effective length of the gas jet determined by the nozzle SPIG distance to $12 \mathrm{~mm}$ in on-line operation, which is still larger than the laser beam diameter of $5 \mathrm{~mm}$ realized at the experiment.

A second test was performed in which we investigated the replacement of the straight SPIG with an inner diameter of
$3 \mathrm{~mm}$, a rod diameter of $1.5 \mathrm{~mm}$ and a length of $126 \mathrm{~mm}$ [18] by a bell-shaped SPIG. Here the inner diameter towards the gas cell is increased to about $6 \mathrm{~mm}$ which then smoothly reduces to the normal inner diameter of $3 \mathrm{~mm}$. This geometry is sketched in the green encircled area in Fig. 1 and was intended to improve the ion extraction. Due to the larger acceptance area of the SPIG this geometry was found to be less sensitive to the alignment of the gas cell with the de Laval nozzle, while the total performance in extraction efficiency and in-gas-cell to in-gas-jet ionization was comparable to a well aligned normal SPIG geometry. A clear disadvantage precluding the bell-shaped SPIG from further considerations was the reduced differential pumping capability. The pressure exceeded $1 \cdot 10^{-4}$ mbar in the extraction chamber at the nominal gas cell pressure of 350 mbar for all distances, indicating that the gas jet is well captured.

Another study during these tests was to investigate the formation of molecular ions after the laser ionization of copper atoms in the gas jet environment. It is known that the atomic ions created in the gas cell can form molecular ions with impurity molecules present in the buffer gas. Most of these ions can be cracked by collision-induced-dissociation (CID) when applying a sufficient voltage between the gas cell and the SPIG [19]. Fig. 3 shows the mass spectra obtained for in-gas-cell ionization and in-gas-jet ionization and the additional effect of a dissociation voltage for the latter. It is clearly visible that argon clusters and nitrogen compounds are still formed in the gas jet while in the gas cell significant amounts of water compounds can be additionally found. This observation can be explained by the difference in gas pressure and thus in the change in total ion-neutral collision rate which is orders of magnitude reduced in the gas jet environment and will therefore decrease the number of molecular loss channels in comparison to the gas cell. This observation is in agreement with earlier measurements on the time structure of different molecular compounds indicating that the formation of argon clusters has the fastest time scale [20]. Nevertheless the formation of clusters between argon (and possibly nitrogen) and the photo-ions in the low density region of the gas jet is somewhat unexpected and has to be considered in further investigations on the in-gas-jet ionization technique. Applying a voltage of $300 \mathrm{~V}$ for the in-jet ionization, most but not all clusters can be dissociated and the abundance of copper ions bound in molecular sidebands is reduced from $46 \%$ to $19 \%$. In addition, the time structures of the ions from the different ionization regions have been measured and conform with the results from previous investigations considering in-SPIG ionization [21].

\section{Laser system}

Efficient and selective laser ionization of short-lived species in the gas jet requires a high-repetition rate laser system, which in addition must include one laser with a narrow bandwidth matching the reduced Doppler broadening in the gas jet to gain in spectral resolution. A high-repetition rate Titanium:sapphire (Ti:Sa) laser system with a broad bandwidth has been installed temporarily at LISOL in 2011 to test the performance for in-gas-cell laser ionization [22]. It was shown that at repetition rates of $10 \mathrm{kHz}$ a similar in-gas-cell ionization efficiency was obtained compared to the Excimer pumped dye laser system operated at $200 \mathrm{~Hz}$ but at higher pulse energies.

For the in-gas-jet laser ionization and spectroscopy of the neutron-deficient actinium isotopes a similar solid-state laser system was commissioned at LISOL in a joint collaboration between GANIL [23], Mainz University [24] and University of Jyväskylä [25]. The layout of the laser system is schematically shown in Fig. 4. It consists of a Nd:YAG pump laser (DM-YAG 60-532, Pho- 


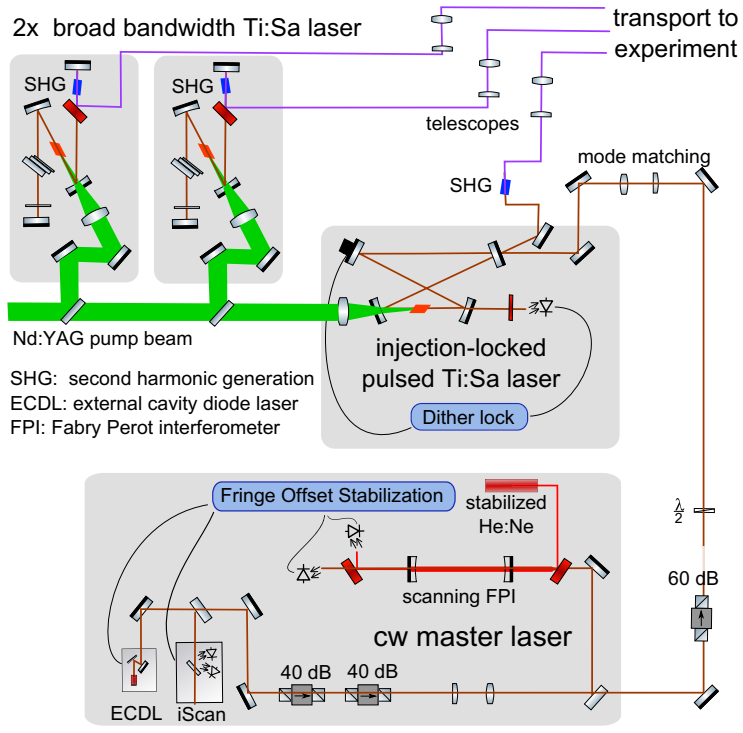

Fig. 4. Schematic setup of the laser system consisting of two broad bandwidth pulsed Ti:Sa lasers and a narrow bandwidth injection-locked Ti:Sa laser. The narrow bandwidth laser is seeded by a cW ECDL as master laser. Additionally, the components for the active stabilization with a He:Ne are shown, see text for details.

tonics Industries Inc.) with an average output power up to $60 \mathrm{~W}$ at $532 \mathrm{mn}$ operated at a repetition rate of $10 \mathrm{kHz}$. This light was used to simultaneously pump three tunable Ti:Sa lasers of which two have a $Z$-shaped resonator featuring a broad bandwidth of about $4 \mathrm{GHz}$ and a pulse length of $35 \mathrm{~ns}$ as used for resonant ionization laser ion sources [24]. They can be tuned in the range of 690$990 \mathrm{~nm}$ (fundamental output) with extension to shorter wavelength by generation of higher harmonics. One of the lasers is used to excite the $424 \mathrm{~nm}$ transition into an AI resonance as shown in the ionization scheme in Fig. 5. The other laser is operated at $438 \mathrm{~nm}$ for a broadband first step excitation but also can be operated at $434 \mathrm{~nm}$ to increase the ionization efficiency by exciting a second $\mathrm{AI}$ transition. As the required laser light is in the blue wavelength region around $430 \mathrm{~nm}$, the broad bandwidth lasers have been operated with intra-cavity second harmonic generation (SHG) [26]. In this method all resonator mirrors are highly reflective for the fundamental laser radiation and the optical non- linear beta barium borate (BBO) crystal used for SHG is installed inside the cavity. This allows an increase of the conversion efficiency as the cavity enhancement of the fundamental radiation can be efficiently used. The second harmonic is coupled out using a dichroic mirror inside the cavity. This configuration generated up to $1.8 \mathrm{~W}$ of laser light in the $430 \mathrm{~nm}$ region using a pump power of about $14.5 \mathrm{~W}$.

The third laser, pumped by the same Nd:YAG laser, was a pulsed narrow bandwidth Ti:Sa laser with a bow-tie geometry. This laser was developed and operated off-line $[27,11]$ but so far never used for on-line operation. The cavity was injection-locked by narrow bandwidth light from a cw external cavity diode laser (ECDL) seeding the pulsed laser radiation at $877 \mathrm{~nm}$. A home-built ECDL in Littrow geometry was used with an anti-reflection (AR) coated laser diode (EYP-RWE-0860, Eagleyard Photonics) for an extended scanning range. Frequency stabilization and control was achieved using a fringe-off-set lock technique. A homemade scanning Fabry-Perot Interferometer (sFPI) with a free spectral range (FSR) of 299.979 MHz - referenced to a stabilized He:Ne laser (SLO3, SIOS Messtechnik) for relative and stable frequency control - was used in combination with a commercial quadrature interferometer stabilization (iScan, TEM Messtechnik) for short-term stabilization. This provided laser light with a bandwidth of a few $\mathrm{MHz}$ which could be scanned over several GHz [28]. To protect the diode laser from back reflections, as well as from the pulsed laser radiation, a total of $140 \mathrm{~dB}$ of optical isolation was installed using three optical Faraday isolators. Due to the stabilization, monitoring and protection measures a laser power of only $6 \mathrm{~mW}$, from the original $26 \mathrm{~mW}$ measured directly after the diode laser, was available for seeding the pulsed Ti:Sa laser. Nevertheless, this power was sufficient to stabilize the cavity and seed the wavelength of the pulsed Ti:Sa laser. After mode matching, the cw laser light was injected into the bow-tie Ti:Sa ring resonator which itself was stabilized onto the diode laser radiation using a dither lock (LaseLock, TEM Messtechnik). As the fundamental wavelength of $877 \mathrm{~nm}$ is relatively far off the Ti:Sa gain maximum around $800 \mathrm{~nm}$, an appropriate mirror set with high reflectivity (HR) coating from 850 to $950 \mathrm{~nm}$ was used to suppress self stimulated lasing at wavelengths possessing a higher gain. In total about $1.5 \mathrm{~W}$ of fundamental laser radiation with a spectral band width of about $10-20 \mathrm{MHz}$, which is a typical value for this laser [11], was available resulting in about 80-100 $\mathrm{mW}$ at $438 \mathrm{~nm}$ after external single pass SHG.
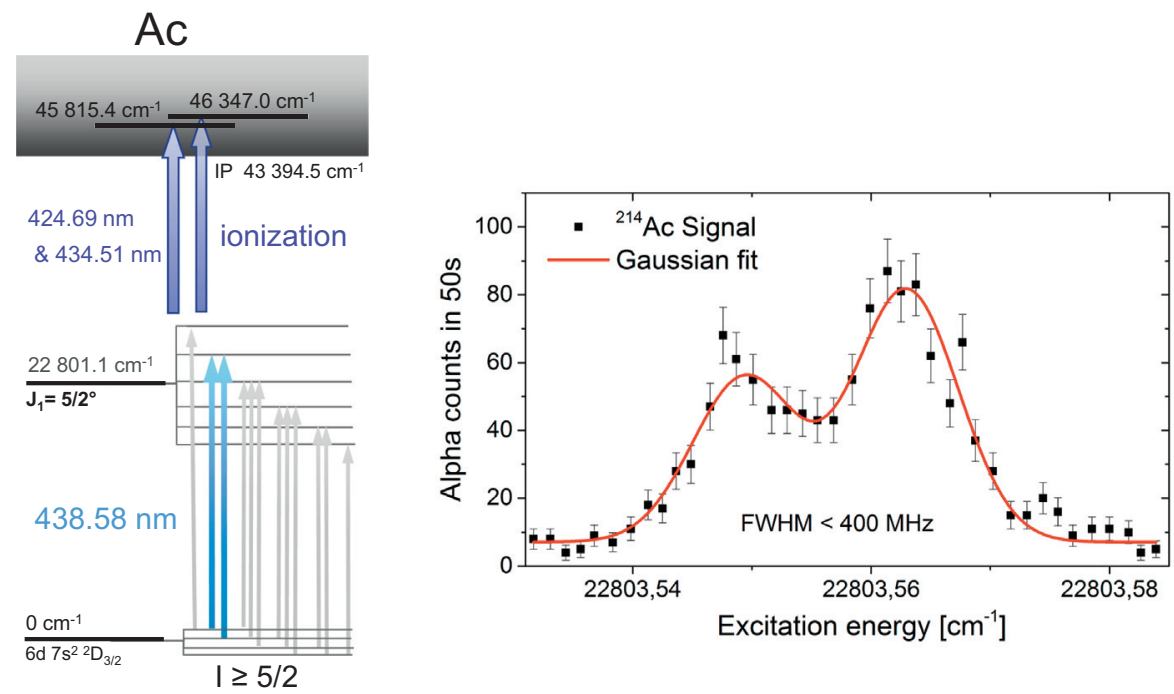

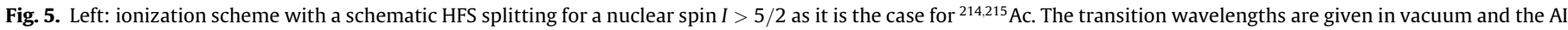
states have been identified in a previous work [7]. Right: Observed signal for two partially resolved HFS transitions as indicated in the ionization scheme in ${ }^{214}$ Ac. 

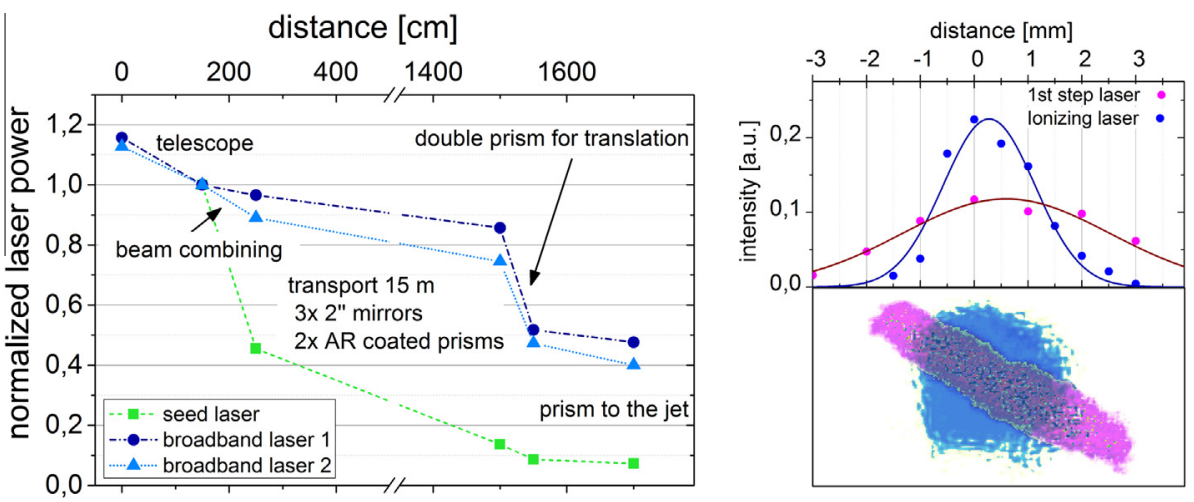

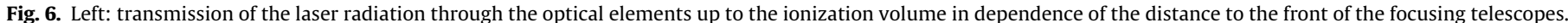

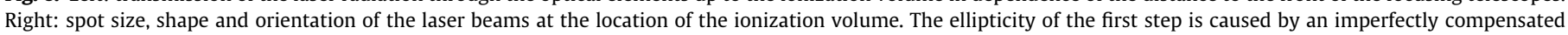
astigmatism from the external SHG, while the orientation is due to the beam transport.

The laser radiation of all three lasers was then shaped using telescopes and guided towards the gas cell located $17 \mathrm{~m}$ away from the laser table. For transport 2" high-reflecting dielectric mirrors and anti-reflection coated quartz prisms were used. After the experiment and all corresponding optimization procedures, the transmission of the laser radiation was measured as shown in the left part of Fig. 6. The ionization lasers show a rather steep drop in transmitted power at a distance of around $15 \mathrm{~m}$ where a pair of prisms was mounted on a translation stage allowing a remote horizontal tuning of all three lasers simultaneously. Unfortunately, it was set up close to the limit of total internal reflection and optimization during the experiment resulted in additional losses, which have to be taken into account when estimating the efficiency and saturation powers. The first step shows significant losses during laser beam transport which is attributed to the imperfectly compensated astigmatism introduced by the external SHG. As the laser power was more than sufficient for spectroscopy and even additional attenuation of a factor of ten was required, this loss in laser power was not further optimized but the quantification is important for reporting the applied laser pulse energies. The right part of Fig. 6 shows the final laser beam size of the first and second step at the gas jet position, thus defining the ionization volume. The upper graph shows the measured profiles projected in one dimension. The lower picture shows the colored and superimposed photographs of each step, but due to saturation of the camera the pictures serves only as a guide to estimate the size and the shape of the laser beam. The elliptical shape of the first step is due to the insufficient astigmatic compensation and will reduce the effective ionization region further. The beam spot width of about $4 \mathrm{~mm}$ is much smaller than the $55 \mathrm{~mm}$ required for the illumination of all atoms in the gas jet at a laser repetition rate of $10 \mathrm{kHz}$. Therefore, a geometrical factor of about $7 \%$ has to be considered for the total efficiency.

\section{Results and outlook}

With the discussed improvements at the gas cell setup and the high-repetition rate laser system it was possible to resonantly ionize on-line produced neutron-deficient actinium isotopes in the gas jet for the first time. Fig. 5 shows a partially resolved spectrum of two HFS transitions observed in ${ }^{214} \mathrm{Ac}$ as indicated in the depicted ionization scheme. The resonances feature a full width half maximum below $400 \mathrm{MHz}$ and the shape is dominated by a Gaussian distribution, which shows that the pressure broadening becomes negligible. In this frequency scan the power of the first step laser was attenuated to $8 \mathrm{~mW}$ on the laser table to avoid saturation broadening and corresponds to about $80 \mathrm{~nJ}$ pulse energy in the ionization region. The total efficiency of this first on-line use of the in-gas-jet technique seems to follow the trend observed in the off-line tests. An evaluation of the efficiency as well as of the spectroscopic results is ongoing.

\section{Acknowledgments}

This work was supported by FWO-Vlaanderen (Belgium), by GOA/2010/010 (BOF KU Leuven), by the IAP Belgian Science Policy (BriX network P7/12) and by a Grant from the European Research Council (ERC-2011-AdG-291561-HELIOS). S.S acknowledges a Ph. D. Grant of the belgian Agency for Innovation by Science and Technology (IWT). L.P.G. acknowledges FWO-Vlaanderen (Belgium) as an FWO Pegasus Marie Curie Fellow.

\section{References}

[1] T. Cocolios, A. Andreyev, B. Bastin, N. Bree, J. Büscher, J. Elseviers, J. Gentens, M. Huyse, Yu. Kudryavtsev, D. Pauwels, et al., Phys. Rev. Lett. 103 (2009) 102501, http://dx.doi.org/10.1103/PhysRevLett. 103.102501.

[2] R. Ferrer, N. Bree, T. Cocolios, I. Darby, H. De Witte, W. Dexters, J. Diriken, J. Elseviers, S. Franchoo, M. Huyse, et al., Phys. Lett. B 728 (2014) 191-197, http://dx.doi.org/10.1016/j.physletb.2013.11.055.

[3] Yu. Kudryavtsev, R. Ferrer, M. Huyse, P. Van den Bergh, P. Van Duppen, Nucl Instr. Meth. B 297 (2012) 7-22, http://dx.doi.org/10.1016/j.nimb.2012.12.008.

[4] Yu. Kudryavtsev, P. Creemers, R. Ferrer, C. Granados, L. Gaffney, M. Huyse, E. Mogilevskiy, S. Raeder, S. Sels, P. Van den Bergh, P. Van Duppen, A. Zadvornaya, Nucl. Instr. Meth. B 376 (2016) 345-352.

[5] R. Ferrer, B. Bastin, D. Boilley, P. Creemers, P. Delahaye, E. Liénard, X. Fléchard, S. Franchoo, L. Ghys, M. Huyse, et al., Nucl. Instr. Meth. B 317 (2013) 570-581, http://dx.doi.org/10.1016/j.nimb.2013.07.028.

[6] I. Ahmad, P. Butler, Annu. Rev. Nucl. Part. Sci. 43 (1993) 71-116, http://dx.doi. org/10.1146/annurev.ns.43.120193.000443.

[7] S. Raeder, M. Dombsky, H. Heggen, J. Lassen, T. Quenzel, M. Södin, A Teigelhöfer, K. Wendt, Hyperfine Interact. 216 (2013) 33-39, http://dx.doi. org/10.1007/s10751-013-0832-7.

[8] W. Meggers, Spectrochim. Acta 10 (1957) 195-200, http://dx.doi.org/10.1016/ 0371-1951(57)80186-6.

[9] W. Meggers, M. Fred, F. Tomkins, J. Res. Natl. Bur. Stand. 58 (1957) 297-315, http://dx.doi.org/10.6028/jres.058.037.

[10] J. Roßnagel, S. Raeder, A. Hakimi, R. Ferrer, N. Trautmann, K. Wendt, Phys. Rev A 85 (2012) 012525, http://dx.doi.org/10.1103/PhysRevA.85.012525.

[11] V. Sonnenschein, Laser developments and high resolution resonance ionization spectroscopy of actinide elements (Ph.d. thesis), Jyväskylä, 2014. ISBN 978-951-39-6050-6.

[12] C. Granados, et al. (2015), in preparation.

[13] A. Andreyev, D. Bogdanov, V. Chepigin, A. Kabachenko, O. Malyshev, Y.A Muzychka, B. Pustylnik, G. Ter-Akopian, A. Yeremin, Nucl. Phys. A 568 (1994) 323-332, http://dx.doi.org/10.1016/0375-9474(94)90205-4.

[14] Yu. Kudryavtsev, R. Ferrer, M. Huyse, P. Van den Bergh, P. Van Duppen, L. Vermeeren, Rev. Sci. Instrum. 85 (2013) 02B915, http://dx.doi.org/10.1063/ 1.4850695 .

[15] L. Vermeeren, N. Bijnens, M. Huyse, Yu. Kudryavtsev, P. Van Duppen, J. Wauters, Z. Qamhieh, P. Thoen, E. Vandeweert, R. Silverans, Phys. Rev. Lett. 73 (1994) 1935, http://dx.doi.org/10.1103/PhysRevLett. 73.1935. 
[16] Yu. Kudryavtsev, T.E. Cocolios, J. Gentens, M. Huyse, O. Ivanov, D. Pauwels, T. Sonoda, P. Van den Bergh, P. Van Duppen, Nucl. Instr. Meth. B 267 (2009) 2908-2917, http://dx.doi.org/10.1016/j.nimb.2009.06.013.

[17] Yu. Kudryavtsev, B. Bruyneel, S. Franchoo, M. Huyse, J. Gentens, K. Kruglov, W. Mueller, N. Prasad, R. Raabe, I. Reusen, et al., Nucl. Phys. A 701 (2002) 465-469, http://dx.doi.org/10.1016/S0375-9474(01)01628-1.

[18] P. Van den Bergh, S. Franchoo, J. Gentens, M. Huyse, Yu. Kudryavtsev, A Piechaczek, R. Raabe, I. Reusen, P. Van Duppen, L. Vermeeren, A. Wöhr, Nucl. Instr. Meth. B 126 (1997) 194-197, http://dx.doi.org/10.1016/S0168-583X(96) 01011-7.

[19] Yu. Kudryavtsev, B. Bruyneel, M. Huyse, J. Gentens, P. Van den Bergh, P. Van Duppen, L. Vermeeren, Nucl. Instr. Meth. B 179 (2001) 412-435, http://dx.doi. org/10.1016/S0168-583X(01)00575-4.

[20] Yu. Kudryavtsev, M. Facina, M. Huyse, J. Gentens, P. Van den Bergh, P. Van Duppen, Nucl. Instr. Meth. B 204 (2003) 336-342, http://dx.doi.org/10.1016/ S0168-583X(02)01946-8.

[21] T. Sonoda, T. Cocolios, J. Gentens, M. Huyse, O. Ivanov, Yu. Kudryavtsev, D. Pauwels, P. Van den Bergh, P. Van Duppen, Nucl. Instr. Meth. B 267 (2009) 2918-2926, http://dx.doi.org/10.1016/j.nimb.2009.06.085.
[22] R. Ferrer, V. Sonnenschein, B. Bastin, S. Franchoo, M. Huyse, Yu. Kudryavtsev, T. Kron, N. Lecesne, I. Moore, B. Osmond, et al., Nucl. Instr. Meth. B 291 (2012) 29-37, http://dx.doi.org/10.1016/j.nimb.2012.08.023.

[23] N. Lecesne, R. Alves-Conde, E. Coterreau, F. De Oliveira, M. Dubois, J.L. Flambard, H. Franberg, T. Gottwald, P. Jardin, J. Lassen, F. Le Blanc, R. Leroy, C. Mattolat, A. Olivier, J.Y. Pacquet, A. Pichard, S. Rothe, M.G. Saint-Laurent, K. Wendt, Rev. Sci. Instrum. 81 (2010), http://dx.doi.org/10.1063/1.3279301.

[24] C. Mattolat, S. Rothe, F. Schwellnus, T. Gottwald, S. Raeder, K. Wendt, A.I.P. Conf. Proc. 1104 (2009) 114, http://dx.doi.org/10.1063/1.3115586.

[25] M. Reponen, I.D. Moore, T. Kessler, I. Pohjalainen, S. Rothe, V. Sonnenschein, Eur. Phys. J. A 48 (2012), http://dx.doi.org/10.1140/epja/i2012-12045-2.

[26] V. Sonnenschein, I.D. Moore, I. Pohjalainen, M. Reponen, S. Rothe, K. Wendt, J.P. S. Conf. Proc. 6 (2015) 030126, http://dx.doi.org/10.7566/JPSCP.6.030126.

[27] T. Kessler, H. Tomita, C. Mattolat, S. Raeder, K. Wendt, Laser Phys. 18 (2008) 842-849, http://dx.doi.org/10.1134/S1054660X08070074.

[28] A. Hakimi, Diodenlaserbasierte Resonanzionisations-Massenspektrometrie zur Spektroskopie und Ultraspurenanalyse an Uranisotopen (Ph.d. thesis), Mainz, 2014. URN: urn:nbn:de:hebis:77-35106. 